\title{
Erratum: Nuclear axial currents in chiral effective field theory [Phys. Rev. C 93, 015501 (2016)]
}

\author{
A. Baroni, L. Girlanda, S. Pastore, R. Schiavilla, and M. Viviani
}

(Received 17 March 2017; published 12 May 2017)

DOI: 10.1103/PhysRevC.95.059901

After the authors of Ref. [1] pointed out the incorrect chiral behavior of one of the loop corrections to the axial current ( $\left.\mathbf{j}_{5, a}\right)$, we have reexamined the derivation of all these corrections in our formalism. As a result, an error was found in the loop function $W_{3}(k)$ first given in Eq. (D12) of the original paper and its earlier Erratum [2]. The correct expression is

$$
W_{3}(k)=-\frac{1}{2} \int_{0}^{1} d z \frac{1}{M(k, z)},
$$

which is finite in the chiral limit. The error can be traced back to Eqs. (5.11) and (D4) of the original paper and its earlier Erratum [2], which should have read

$$
\begin{aligned}
\mathbf{j}_{5, a}^{(1)}(\mathrm{e} 8)= & -\frac{g_{A}^{5}}{16 f_{\pi}^{4}}\left(\tau_{2, a}\left\{\left(\sigma_{1} \times \mathbf{k}_{2}\right) \times \mathbf{k}_{2}\left[k_{2}^{2} S^{(0)}\left(k_{2}\right)-S^{(2)}\left(k_{2}\right)\right]+\left[k_{2}^{2} S^{(2)}\left(k_{2}\right)-S^{(4)}\left(k_{2}\right)\right] \sigma_{1}-\left[k_{2}^{2} S_{i j}^{(2)}\left(\mathbf{k}_{2}\right)-S_{i j}^{(4)}\left(\mathbf{k}_{2}\right)\right] \sigma_{1 j}\right\}\right. \\
& \left.-4 \tau_{1, a} \epsilon_{i j k} k_{2 j} S_{k l}^{(2)}\left(\mathbf{k}_{2}\right)\left(\sigma_{2} \times \mathbf{k}_{2}\right)_{l}\right),
\end{aligned}
$$

and

$$
\begin{aligned}
\mathbf{j}_{5, a}^{(1)}(\mathrm{e} 8)= & -\frac{g_{A}^{5}}{64 \pi f_{\pi}^{4}} \int_{0}^{1} d z\left\{\tau _ { 2 , a } \left(5 \boldsymbol{\sigma}_{1} M\left(k_{2}, z\right)+\frac{\mathbf{k}_{2}}{2} \boldsymbol{\sigma}_{1} \cdot \mathbf{k}_{2}\left[\frac{k_{2}^{2}(z \bar{z})^{2}}{M\left(k_{2}, z\right)^{3}}+\frac{1-7 z \bar{z}}{M\left(k_{2}, z\right)}\right]\right.\right. \\
& \left.\left.+\frac{k_{2}^{2}}{2} \boldsymbol{\sigma}_{1}\left[\frac{9 z \bar{z}-1}{M\left(k_{2}, z\right)}-\frac{k_{2}^{2}(z \bar{z})^{2}}{M\left(k_{2}, z\right)^{3}}\right]\right)+\frac{\tau_{1, a}}{2}\left(\boldsymbol{\sigma}_{2} \times \mathbf{k}_{2}\right) \times \mathbf{k}_{2} \frac{1}{M\left(k_{2}, z\right)}\right\},
\end{aligned}
$$

respectively. None of the other results and conclusions are affected by this error. This error propagated into a subsequent paper [3] and an Erratum relative to this second paper Ref. [4].

[1] H. Krebs, E. Epelbaum, and U.-G. Meißner, Ann. Phys. 378, 317 (2017).

[2] A. Baroni, L. Girlanda, S. Pastore, R. Schiavilla, and M. Viviani, Phys. Rev. C 93, 049902(E) (2016).

[3] A. Baroni, L. Girlanda, A. Kievsky, L. E. Marcucci, R. Schiavilla, and M. Viviani, Phys. Rev. C 94, 024003 (2016).

[4] A. Baroni, L. Girlanda, A. Kievsky, L. E. Marcucci, R. Schiavilla, and M. Viviani, following Erratum, Phys. Rev. C 95, 059902 (2017). 